\title{
The Use of Mind-body Medicine in Chronic Pain Management: Differential Trends and Session-by-Session Changes in Anxiety
}

\section{David Cosio ${ }^{1^{\star}}$ and Sujata Swaroop ${ }^{2}$}

${ }^{1}$ Anesthesiology/Pain Clinic, Jesse Brown VA Medical Center, USA

${ }^{2}$ Pain Clinic/Psychology, Jesse Brown VA Medical Center, USA

\begin{abstract}
The evidence to date suggests that the use of mind-body medicine in chronic pain management can improve physical and psychological symptoms. However, past research evidence has largely relied on global measures of distress at pre- and post-intervention. Even though it is plausible that reported anxiety occurs in the context of pain, there is also evidence to suggest a reciprocal relationship. Thus, the purpose of the current study was to determine the differential impact that mind-body medical interventions have on anxiety among Veterans with chronic, non-cancer pain. The current study utilized multiple, repeated assessments of anxiety to better understand changes made over time between two mind-body interventions (Acceptance and Commitment Therapy (ACT) and Cognitive Behavioral Therapy (CBT)) used for chronic pain management. Ninety-six Veterans elected to participate in either intervention following the completion of a pain health education program at a Midwestern VA Medical Center between November 3, 2009-November 4, 2010. A $2 \times 7$ repeated measures multivariate analyses of variance indicated significantly lower levels of global distress by the end of both the ACT and CBT interventions. Trend analysis revealed differential patterns of change in levels of anxiety over time. Helmert contrast analyses found several modules of ACT were statistically different than the overall mean of previous sessions. Implications related to timing and patterns of change for the interventions are discussed.
\end{abstract}

Keywords: Acceptance and commitment therapy; Cognitivebehavioral therapy; Chronic pain; Trend analysis; Brief therapy; Beck anxiety inventory

\section{Introduction}

A positive relationship exists between pain and anxiety in clinical settings. Past research has found these associations to be larger than those between pain and depression [1]. In fact, a recent study found that relief from anxiety and low baseline depression were the most important predictors for pain relief and the most strongly associated with functional improvement [2]. Past research has also shown that the strongest associations between pain and anxiety were observed with panic and post-traumatic stress disorders as defined by the DSMIVR [3]. These findings suggest there needs to be an improved effort regarding the detection and treatment of anxiety disorders in pain treatment settings. The human experience of pain is not merely somatic, but it also varies according to mental, emotional, and physical factors that work via similar mechanisms and have synergistic effects [4]. For any two conditions, there are several possible relationship scenarios. Even though it is plausible that reported anxiety occurs in the context of pain, there is also evidence to suggest a reciprocal relationship [5]. The mutual maintenance model holds that anxiety maintains or exacerbates symptoms of pain, and vice versa [4,6]. Anxiety levels have been shown to predict pain severity and behaviour in patients who suffer from chronic pain [7]. Certain signs of anxiety, such as catastrophizing and hypervigilance, have been shown to play key roles in chronic pain. Thus, comorbid anxiety has implications for the impact and outcome of chronic pain [5].

Mind-body medicine emphasize in engaging both in mind and body to promote stress reduction and well-being by changing the manner in which patients respond to their stressors. Any intervention that changes a patient's mental or emotional state will produce corresponding changes in the body and could therefore be called a "mind-body" intervention [1]. Thus, mind-body therapies can also be used to treat and/or prevent a variety of conditions, including chronic pain disorders [8]. Some of the most commonly used therapies include mindfulness, as practiced in Acceptance and Commitment Therapy (ACT), and relaxation techniques, as trained in traditional CognitiveBehavioural Therapy (CBT). Both ACT [9-13] and CBT [14-21] have been recognized in past literature as empirically supported treatments for chronic pain. In addition, research has provided evidence that ACT compares favourably with traditional CBT in the treatment of chronic pain [22-25]. Recently there has been some debate about utilizing global measures of distress as pre- and post-assessments for chronic pain treatment, given its multiple dimensions [26,27]. Unfortunately, current literature lacks consensus for guiding clinicians regarding selection of these measures. For example, the Beck Anxiety Inventory ${ }^{\circ}$ (BAI) [28] has been identified as a measure of pain-related anxiety [29], but other scholars warn there may be potential for misinterpretation of anxiety symptoms as symptoms of pain [30]. However, utilizing this measure for multiple, repeated assessments of anxiety may serve as corroboration to pre- and post-assessments when measuring distress [31]. Furthermore, assessing change session-by-session may allow future modifications to be made to enhance results, give therapists a better understanding of effective techniques, and provide evidence of treatment effectiveness to stakeholders [32].

Pain is one of the most common reasons Veterans consult with their primary care providers [33]. In fact, about half of the Veterans within the

*Corresponding author: David Cosio, Anesthesiology/Pain Clinic \#124, Jesse Brown VA Medical Center, Chicago, IL 60612, USA, Tel: (312)569-8703; Fax: (312)569-8120; E-mail: david.cosio2@va.gov

Received March 03, 2016; Accepted March 26, 2016; Published March 30 2016

Citation: Cosio D, Swaroop S (2016) The Use of Mind-body Medicine in Chronic Pain Management: Differential Trends and Session-by-Session Changes in Anxiety. J Pain Manage Med 2: 114.

Copyright: $\odot 2016$ Cosio D, et al. This is an open-access article distributed under the terms of the Creative Commons Attribution License, which permits unrestricted use, distribution, and reproduction in any medium, provided the original author and source are credited. 
VA experience pain regularly [34]. Veterans with chronic pain are often more complex than civilians due to difficulties returning to their private life and the influence of their past military service on their pain [35]. Previous research has found that past military service may contribute to hypersensitivity [36-38], and may serve as an exemplar population to determine the efficacy of mind-body medical interventions for the treatment of physiological and psychological distress. In 2009, the VA advocated for the "Stepped Care Model of Pain Management" as a best practice model [39]. The stepped care model gives providers the ability to escalate treatment options to include specialized care and multidisciplinary approaches (Figure 1). The purpose of the current study was to determine the differential impact that mind-body medical interventions have on psychological distress among Veterans with chronic, non-cancer pain within this framework. To this end, change in distress across the two treatment conditions was measured using multiple assessments of anxiety over many weeks, allowing the examination of different patterns over the course of treatment.

\section{Materials and Methods}

\section{Participants}

Two hundred and six Veterans were introduced to the mind-body medical interventions while participating in a pain health education program during Step \#2 of the care model [40]. One hundred and ten Veterans elected to participate in either intervention following the completion of the pain health education program at a Midwestern VA Medical Center between November 3, 2009-November 4, 2010. The current study had no exclusion criteria in order to reflect the population as it is encountered in clinical practice.

\section{Procedure}

Potential candidates were then scheduled for the next available selected intervention. Groups have been shown to be useful in breaking down isolation, enhancing motivation, and providing the benefits of learning from the insights and experiences of other group members. In addition, a recent literature review of various aspects of delivering psychosocial interventions for chronic pain found that group-delivered courses showed more beneficial effects [41]. Thus, each Veteran was subsequently scheduled for 10-weeks of one-hour, ACT or brief CBT

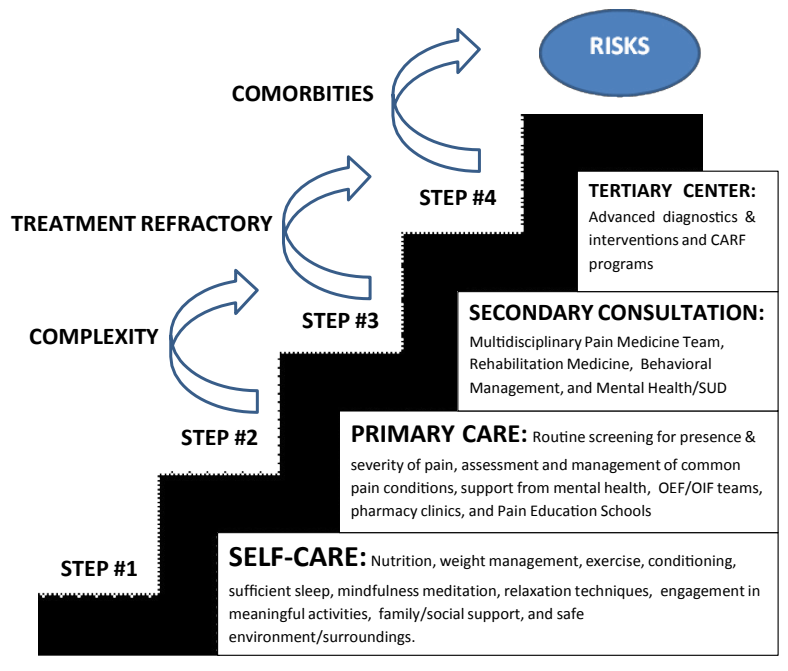

Figure 1: The VA's stepped care model of pain management. group sessions. Enrollment in each group was capped at 15 Veterans per cohort. Participants were seen on a weekly or biweekly basis depending if there was a holiday scheduled. Therapists in this study were licensed clinical psychologists coupled with advanced graduate trainee therapists who had been trained to work with Veterans. Prior research has supported the use of graduate trainees in mind-body medical interventions [42]. Veterans voluntarily participated in the program and were free to withdraw at any time. Veterans were given free parking validation/transportation reimbursement if they were in attendance and were qualified for such programs. The current study's protocol was reviewed and approved by the affiliated university's Institutional Review Board and the VA's Research and Development office. A wavier of informed consent was granted due to the retrospective nature of the study and the minimal risk to subjects who participated.

\section{Mind-body interventions}

Cognitive-behavioral therapy (CBT): CBT is a structured, time-limited, present-focused approach to psychotherapy that helps patients develop strategies to modify dysfunctional thinking patterns and maladaptive behaviors in order to assist them in resolving current problems. The 10-week CBT treatment group was a brief protocol based on an empirically supported, "Treatments That Work" manual [43], and sessions addressed different ways to cope with and reduce experience of chronic pain (Table 1). The protocol reviews pain education topics, introduces cognitive concepts, and teaches behavioural strategies. CBT therapy for chronic pain has been disseminated nationwide by the VA Office of Mental Health Services and National Pain Management Program Office.

Acceptance and commitment therapy (ACT): ACT is distinct from other mindfulness-based interventions, such as MindfulnessBased Stress Reduction (MBSR), Mindfulness-Based Cognitive Therapy (MBCT), and Dialectical behaviour therapy (DBT). ACT is a form of clinical behaviour analysis employed in psychotherapy that uses acceptance and mindfulness strategies mixed with commitment and behaviour-change strategies to increase psychological flexibility. MBSR uses a combination of mindfulness meditation, body awareness, and yoga to help people become more mindful. MBCT uses traditional CBT methods and adds in mindfulness meditation. Mindfulness is

\begin{tabular}{|c|c|c|c|c|}
\hline S. No. & $\begin{array}{l}\text { Cognitive-behavioral } \\
\text { therapy protocol }\end{array}$ & $\begin{array}{l}\text { Helmert } \\
\text { contrast } \\
\text { P-value }\end{array}$ & $\begin{array}{l}\text { Acceptance and } \\
\text { commitment } \\
\text { therapy protocol }\end{array}$ & $\begin{array}{l}\text { Helmert } \\
\text { contrast } \\
\text { p-value }\end{array}$ \\
\hline 1 & Education on chronic pain & - & Introduction to ACT & - \\
\hline 2 & $\begin{array}{l}\text { Theories of pain and } \\
\text { diaphragmatic breathing }\end{array}$ & - & Controlling your pain & - \\
\hline 3 & $\begin{array}{l}\text { Progressive muscle } \\
\text { relaxation and visual } \\
\text { imagery }\end{array}$ & 0.96 & What do you value? & 0.01 \\
\hline 4 & $\begin{array}{l}\text { Automatic thoughts and } \\
\text { pain; anger management }\end{array}$ & 0.35 & Cognitive defusion & 0.00 \\
\hline 5 & Cognitive restructuring & 0.23 & $\begin{array}{l}\text { Practicing } \\
\text { mindfulness }\end{array}$ & 0.37 \\
\hline 6 & Stress management & 0.37 & $\begin{array}{l}\text { Reaching } \\
\text { acceptance }\end{array}$ & 0.02 \\
\hline 7 & Time-based activity pacing & 0.45 & $\begin{array}{l}\text { Making a } \\
\text { commitment to action }\end{array}$ & 0.05 \\
\hline 8 & $\begin{array}{l}\text { Pleasant activity } \\
\text { scheduling }\end{array}$ & 0.45 & Facing obstacles & 0.00 \\
\hline 9 & Sleep hygiene & - & $\begin{array}{l}\text { Living beyond your } \\
\text { pain }\end{array}$ & - \\
\hline 10 & $\begin{array}{l}\text { Relapse prevention } \\
\text { and flare-up planning; } \\
\text { termination }\end{array}$ & - & Termination & - \\
\hline
\end{tabular}

Table 1: Comparison of the 10-week ACT and traditional CBT protocols. 
a "core" exercise used in DBT, which combines standard cognitive behavioural techniques for emotion regulation with concepts of distress tolerance derived from Buddhist meditative practice. The 10-week ACT treatment group followed an amalgamation of established protocols $[44,45]$ and a self-help workbook [46] in which sessions addressed participants' relationship with their thoughts, feelings, memories, and bodily reactions to pain. The current amalgamated protocol was established and served as a best course of treatment within the ACT model for the chronic pain population (Table 1).

\section{Assessment instruments}

As part of the introduction and conclusion of each intervention, Veterans completed a pre- and post-intervention assessment which included the Numeric Rating Scale (NRS-11) [47] and the Brief Symptom Inventory ${ }^{\circ} 18$ (BSI-18) [48]. Veterans also completed the Beck Anxiety Inventory (BAI) [28] before the beginning of each group session in both interventions.

Numeric rating scale (NRS-11): The NRS-11 is an 11-point scale for patient self-reporting of pain for adults and children over 10 years old. Scores range from 0 to 10 , with " 0 " meaning no pain, " 1 to 3 " mild pain, " 4 to 6 " moderate pain, and " 7 to 10 " severe pain.

Brief symptom inventory ${ }^{\circ}-18$ : The BSI-18 is an 18 -item measure of general psychological distress used with adult medical and community populations. The BSI-18 subjectively measures the participants' degree of global distress. Scores are calculated from responses indicated on a five-point Likert scale. This measure has been shown to have satisfactory reliability for the measure of global distress [48]. The internal consistency for the BSI-18 in the current study was good ( $\alpha=0.84)$. Permission to use the BSI-18 is inherent in the qualified purchase of the test materials.

Beck anxiety inventory : The BAI is a 21 -item measure of anxiety severity used extensively with adults. The BAI subjectively measures how the participant has been feeling in the last week, with each item representing one symptom of anxiety. The BAI functions more adequately in anxiety disorders with a high somatic component, such as panic disorder, and has been shown to be less contaminated by depressive content. A total anxiety score is calculated from responses indicated on a four-point Likert scale. The BAI was chosen based on its utilization to measure anxiety by past pain management investigators $[26,49]$. The BAI is the third most utilized research measure of anxiety [50]. The BAI has demonstrated high internal consistency, with Cronbach's alphas $0.90-0.92$, and satisfactory test-retest reliability, $r=0.75[28,49]$. The internal consistency of the BAI in the current study was good $(\alpha=0.91)$. Permission to use the BAI is inherent in the qualified purchase of the test materials.

\section{Data analyses}

A one-way analysis of variance and independent samples t-tests identified differences on demographic and outcome variables at baseline. The relationship between the BAI and the BSI-18 was measured using Spearman's correlation. The 21 items of the BAI were then subjected to principal components analysis (PCA) followed by a varimax rotation with Kaiser normalization using the SPSS 20 to cross validate the measure's factor structure. A last-observation-carriedbackward approach [51] was used for missing data on items on the preassessment and a last-observation-carried-forward approach [52] was used for missing data on items on the post-assessment. The Power and Sample Size Program [53] was utilized to calculate sample size using an anticipated effect size (Cohen's d) of 0.50 , a desired statistical power level greater than or equal to 0.80 , and a probability level less than or equal to 0.05 . The minimum total sample size (pairs of subject scores) was $\mathrm{N}=33$.

The primary intervention outcome analysis was a $2 \times 7$ repeated measures (RM) multivariate analyses of variance (MANOVA). The $\mathrm{ACT}$ and brief CBT protocols were defined as "Intervention Condition" which served as the between-subjects factor and several weekly assessment points (session \#2- session \#8) were defined as "Time" and served as the within-subjects factor. An efficacy subset analysis strategy was utilized. A trend analysis was then computed to explore the presence and nature of the relationship between "Intervention Condition" and "Time" employing polynomial functions. Furthermore, Helmert contrast analyses were conducted in order to test how each module added to the trend over time.

\section{Results}

\section{Participant characteristics}

Eighty-seven percent $(\mathrm{N}=96)$ of the sample elected to complete the pre- and post-intervention assessments, and only their responses were included in the current study (Figure 2). Veterans had mixed idiopathic chronic, non-cancer pain conditions. Most Veterans were African American (78\%), but 16\% were Caucasian and 6\% identified as being Hispanic/Latino. Most were males (90\%), but there were also a representative sample of female Veterans (10\%). Most of the Veterans were 45 to 54 years old $(40 \%)$ or 55 to 64 years old $(38 \%)$, and the youngest returning Veterans (17-24 years old) were not a represented age group. The reported pain score at baseline for all the Veterans in the current study was " 6.02 ;" at post-intervention the average pain score remained similar "5.82" (moderate pain). The mean for the baseline BSI-18 global distress score was 25.63 ( $\mathrm{SD}=13.06$ ), and the BAI baseline total score was $19.50(\mathrm{SD}=11.23)$, which indicate a moderate level of psychological distress among the Veteran sample. The average ACT group member attended 7 out of 10 sessions $(\mathrm{SD}=1.86)$, while the average brief CBT group member attended 8 out of 10 sessions $(\mathrm{SD}=2.03)$. Five, group offerings of the ACT protocol and four of the brief $\mathrm{CBT}$ protocol were conducted during the time of the investigation.

\section{Differences at baseline}

Independent samples t-tests revealed there was no significant

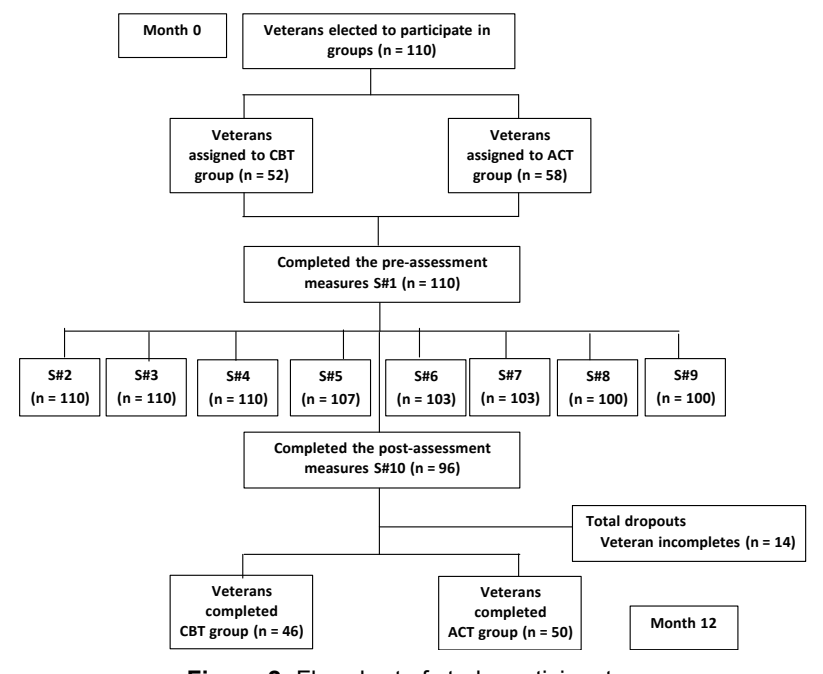

Figure 2: Flowchart of study participants. 
differences between the mind-body medical interventions in reference to members' sex demographic $(p=0.24)$. ANOVA findings indicated there was no differences in group assignment $(p=0.10)$ or race/ethnic demographics $(p=0.71)$ between the interventions. ANOVA also revealed there was no significant difference between the interventions on baseline BSI-18 scores, $\mathrm{F}(1,94)=3.12, p=0.08$, and the baseline BAI total scores, $\mathrm{F}(1,94)=1.04, p=0.31$.

\section{Psychometric outcomes}

The Spearman correlation between the BAI and BSI-18 was $r=0.75, p=0.00$. These findings build upon previous research validating the utility of the BSI-18 [48] and the BAI [28,49,54] as measures of psychological distress among different populations. Prior to performing PCA, the suitability of data for factor analysis was assessed in accordance with previous recommendations [55]. The Kaiser-MeyerOklin value [56,57] was 0.85 and the Barlett's Test of Sphericity [58] reached statistical significance $p=0.00$. PCA revealed the presence of five components with eigenvalues exceeding Kaiser's criterion (Table 2). Only one component had an eigenvalue exceeding the corresponding criterion value for a randomly generated data matrix of the same size [59]. Thus, the current findings support past research which has used the BAI as a unidimensional measure of distress in a pain population [49].

\section{Intervention outcomes}

A $2 \times 7$ RM MANOVA found a significant multivariate "Intervention Condition $\times$ Time" interaction, $\mathrm{F}(6,87)=2.18, p=0.05$, Wilk's Lambda $=0.87$, and a significant main effect for "Time" on the combined set of measures, $\mathrm{F}(6,87)=2.34, p=0.04$, Wilk's Lambda $=0.86$. There was no significant main effect for "Intervention," Wilks' $\lambda=0.95$, $\mathrm{F}(6,87)=0.63, \mathrm{p}=0.73$, which indicates that ACT and brief CBT were not significantly different on their impact on the dependent measures of pain severity and global distress. A significant main effect for time was not obtained for the primary measure of pain severity, $\mathrm{F}(1,94)=1.85$, $p=0.18$. The Mauchly's Sphericity Test result was $0.05(p=0.00)$.

Polynomial functions indicated that the linear $(p=0.04)$ component was significant for "Intervention Condition $\times$ Time." Findings also showed that the quadratic $(p=0.05)$ component was significant for "Time." In accordance with past research, a significant effect meant that the associated line fit the means at better than chance levels [60]. The trend analysis revealed that the groups show different linear trends over time. There was not a significant treatment effect for "Intervention Condition," $\mathrm{F}(1,92)=0.01, p=0.92$.

Helmert contrast analyses did find that the means of several modules of the ACT protocol were statistically different than the overall mean of the previous modules. However, the analyses did not find that the means of any module of the brief CBT protocol were statistically different than the overall mean of the previous modules (Figure 3 ).

\section{Discussion}

While prevailing literature clearly demonstrates the efficacy of

\begin{tabular}{|c|c|c|c|}
\hline $\begin{array}{c}\text { Component } \\
\text { Number }\end{array}$ & $\begin{array}{c}\text { Actual Eigenvalue } \\
\text { from PCA }\end{array}$ & $\begin{array}{c}\text { Criterion Value } \\
\text { from Parallel } \\
\text { Analysis }\end{array}$ & Decision \\
\hline 1 & 8.088 & 1.943 & Accept \\
\hline 2 & 1.611 & 1.769 & Reject \\
\hline 3 & 1.486 & 1.635 & Reject \\
\hline 4 & 1.241 & 1.519 & Reject \\
\hline 5 & 1.097 & 1.406 & Reject \\
\hline
\end{tabular}

Table 2: Components with eigenvalues exceeding Kaiser's criterion.

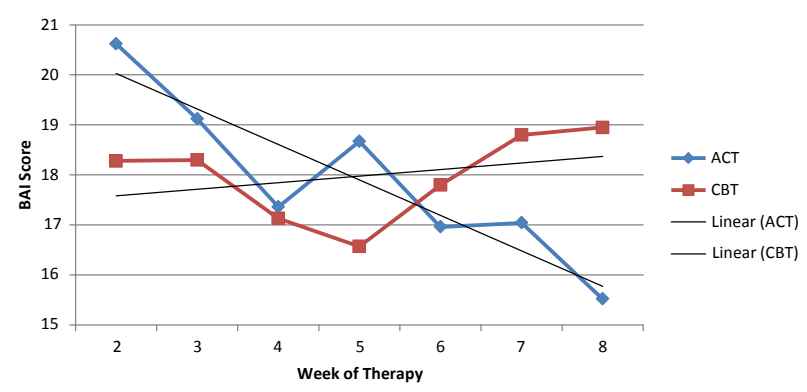

Figure 3: Screeplot for polynomial functions.

mind-body medical interventions for the treatment of psychological and physiological distress, research has traditionally focused on changes in symptoms from pre- to post-treatment [27]. Although establishing efficacy is vital, the bias toward efficacy in extant research has led to the neglect of important questions regarding whether mind-body medical interventions work because of the mechanisms specified by theory [61]. Some researchers have proposed measuring change across therapies in order to improve treatment, to enhance clinical science, and to provide accountability. The current study examined which elements of mindbody medical interventions are contributing to decreased psychological and, in turn, physiological distress, and when change is occurring by measuring change session-by-session in a pain treatment setting. The current study did not find any significant difference over time in pain severity in either mind-body medical intervention. These findings are inconsistent with past research $[10,12,13,25]$. Perhaps, Veterans who suffer from chronic pain may be a unique group of individuals due to the dualism of active duty and civilian life [25]. These compounded experiences may maintain pain at a moderate level (" $4-6$ " on NRS-11). The current Veteran sample reported moderate pain scores at preand post-assessment, which supports this notion. Furthermore, the permanence of chronic pain may undermine recovery and remove a sense of hope or optimism. Therefore, a better adjustment to continuing pain may prove to be a more realistic goal. Thus, further exploration of psychological distress was warranted.

More specifically, this study found a linear trend for Veterans engaged in the ACT intervention group, reflecting a steady decrease in reported distress through sessions focused on learning about the relationship between psychological inflexibility and pain maintenance; letting go of control; identifying one's values; and learning about cognitive defusion. This could be an illustration of a rapid early response. However, after session \#4, distress patterns rose slightly and did not contribute to a statistical difference when compared to previous modules. The progress then continued to decrease through sessions focused on mindfulness; reaching acceptance using self-as-context; and making a commitment to action. In contrast, the current study found a quadratic trend for Veterans engaged in the CBT intervention group, reflecting non-significant decreases in reported distress through sessions focused on diaphragmatic breathing; progressive muscle relaxation and guided imagery; learning about cognitive errors and the $\mathrm{ABC}$ model; cognitive restructuring; and stress response/stress relief. Again, this could be an illustration of a rapid early response. However, after session \#6, distress patterns increased and remained high through sessions focused on activity pacing and pleasant activity scheduling (Figure 3). Note, the mean distress score at session \#8 for ACT was in the mild range $(\mathrm{BAI}=15)$ but remained in the moderate range $(\mathrm{BAI}=19)$ for the CBT group.

Further review of the polynomial screeplot (Figure 3) revealed 
that the scores on the BAI converged around session \#6 for the two interventions before surging in different directions. Of note, it is at the beginning of session \#6 that Veterans have completed the lesson on cognitive defusion in ACT and cognitive restructuring in brief CBT. $\mathrm{ACT}$ and brief CBT overlap to a large extent in shared techniques and strategies with respect to behavioural interventions, such as exposure exercises, problem solving skills, role playing, etc. The major distinction between the ACT and brief CBT interventions lies within their cognitive strategies, which may provide context for the different trends observed. Thus, the results of the current study are consistent with the notion that substantial changes in outcomes should occur following large changes in the assumed mechanism factors [27]. The core message of the ACT intervention is to teach individuals to defuse and distance themselves from their pain instead of suppressing internal experiences as taught in CBT [62]. Perhaps ACT had a more linear trend because cognitive defusion is less demanding of self-regulatory capacity and therefore able to augment an individual's ability to engage in self-regulation [63]. Perchance, the brief CBT intervention had a more quadratic trend because cognitive restructuring is less adaptive and more cognitively demanding than appraising pain experiences [64]. Despite both ACT and brief CBT using manual-based, empirically supported treatment strategies, the findings of the current study suggests that ACT may be more time efficient than brief CBT.

Several limitations in the current study should be noted. First, Veterans self-selected into either the ACT or the brief CBT intervention group. There was also no control group; thus, treatment effects could not be unambiguously reported according to intervention group. However, pain experts have noted that strong outcomes in patients with long-term and intractable conditions together with a clear pattern of results from consequent assessment measures yields a high likelihood that such findings relate to the specific patterns within the respective intervention [65]. Secondly, a potential problem with the current study is its reliance on self-report measures, which may be subject to social desirability biases. However, utilizing the BAI as a frequent assessment did allow for direct measures conducted closely in time and in situation to the behaviour patterns of interest. According to pain scholars, this may reduce report bias and may reduce the possibility of method effects inflating observed relations [65,66]. Another consideration may be that some changes on the outcome measures were the result of participants engaging in other pain management modalities while in the current study and not from the mind-body medical interventions delineated. Finally, the self-regulation skills and abilities of all Veterans with chronic, non-cancer pain may differ as the current sample was predominately African American and did not include a representative sample of the youngest returning soldiers (18-34 years old) when compared to the typical Veteran profile [67].

\section{Conclusions}

In summary, both mind-body medical interventions for chronic pain in the current study experienced a decrease in Veteran reported anxiety. Overall, the current study provided evidence of the treatment effectiveness of both interventions for chronic pain. Yet multiple assessments over several weeks of each intervention indicated distinctive patterns in such trends. Assessing change session-by-session per intervention suggested future modifications should be made to enhance results. For example, the current study suggests that ACT may be more time efficient then CBT for pain groups. Patients enjoy rapid treatment gains. Time efficiency can improve the credibility of the treatment, increase motivation for further change, and lead to increased cost-effectiveness which could make treatment accessible to more individuals in need of assistance. In addition, weekly assessments may have provided a better understanding of effective techniques. For example, each session of the ACT protocol seems to have contributed to a decrease in anxiety except the module immediately following the introduction of cognitive defusion. Despite the findings that ACT was able to enhance self-regulation more than brief CBT, the concept of cognitive defusion may still prove to be too cognitively demanding for patients with chronic pain and additional support or instruction around this concept may prove beneficial. The current examination of the patterns and timing of change per ACT and brief CBT groups, as measured by session-by-session administrations of the BAI, may assist in improved clinical services and encourage future research in chronic, non-cancer pain treatment among Veterans.

\section{Acknowledgements}

We would like to thank all the veterans who participated in the groups for chronic pain; also the graduate assistants, Bonnie Yap, Genna PopovichHymowitz, Joshua Greco, and Scott Sperling for their support in data collection and administration, and Tracy Schmidt and Susan Payvar, licensed psychologists. We would also like to thank Heather Kim for her biostatistical support. Biostatistical services were funded by the National Center for Advancing Translational Sciences, National Institutes of Health, Grant \#UL1TR000050.

\section{Author Disclosure Statement}

Both authors have nothing to disclose. Dr. Sujata Swaroop is currently completing her post-doc in psychology at the Trauma Center at JRI, Project $\mathrm{REACH}$. Earlier versions of this paper were presented at the national Pain Week 2013 and the American Pain Society 2014 conferences. The earlier versions of this paper were modified to reflect the comments received at the conferences.

\section{References}

1. McWilliams LA, Goodwin RD, Cox BJ (2004) Depression and anxiety associated with three pain conditions: results from a nationally representative sample. Pain 111: 77-83.

2. Fuss I, Angst F, Lehmann S, Michel BA, Aeschlimann A (2014) Prognostic factors for pain relief and functional improvement in chronic pain after inpatient rehabilitation. Clin J Pain 30: 279-285.

3. McWilliams LA, Cox BJ, Enns MW (2003) Mood and anxiety disorders associated with chronic pain: an examination in a nationally representative sample. Pain 106: 127-133.

4. Hassed C (2013) Mind-body therapies--use in chronic pain management. Aust Fam Physician 42: 112-117.

5. Gureje $O$ (2008) Comorbidity of pain and anxiety disorders. Curr Psychiatry Rep 10: 318-322.

6. Sharp TJ, Harvey AG (2001) Chronic pain and posttraumatic stress disorder mutual maintenance? Clin Psychol Rev 21: 857-877.

7. van den Hout JH, Vlaeyen JW, Houben RM, Soeters AP, Peters ML (2001) The effects of failure feedback and pain-related fear on pain report, pain tolerance and pain avoidance in chronic low back pain patients. Pain 92: 247-257.

8. Lee C, Crawford C, Hickey A, Active Self-Care Therapies for Pain (PACT) Working Group (2014) Mind-body therapies for the self-management of chronic pain symptoms. Pain Med 15: S21-S39.

9. Dahl J, Wilson K, Nilsson A (2004) Acceptance and commitment therapy and the treatment of persons at risk for long-term disability resulting from stress and pain symptoms: A preliminary randomized trial. Behav Ther 35: 785-802.

10. Johnston M, Foster M, Shennan J, Starkey NJ, Johnson A (2010) The effectiveness of an Acceptance and Commitment Therapy self-help intervention for chronic pain. Clin J Pain 26: 393-402.

11. McCracken LM, Sato A, Taylor GJ (2013) A trial of a brief group-based form of acceptance and commitment therapy (ACT) for chronic pain in general practice: pilot outcome and process results. J Pain 14: 1398-1406.

12. Vowles KE, McCracken LM (2008) Acceptance and values-based action in chronic pain: a study of treatment effectiveness and process. J Consult Clin Psychol 76: 397-407.

13. Wicksell RK, Melin L, Lekander M, Olsson GL (2009) Evaluating the effectiveness of exposure and acceptance strategies to improve functioning and quality of life in longstanding pediatric pain--a randomized controlled trial. Pain 141: 248-257. 
Citation: Cosio D, Swaroop S (2016) The Use of Mind-body Medicine in Chronic Pain Management: Differential Trends and Session-by-Session Changes in Anxiety. J Pain Manage Med 2: 114.

14. Bradley LA, Young LD, Anderson KO, Turner RA, Agudelo CA, et al. (1987) Effects of psychological therapy on pain behavior of rheumatoid arthritis patients. Treatment outcome and six-month followup. Arthritis Rheum 30: 11051114.

15. Goldenberg DL, Kaplan KH, Nadeau MG, Brodeur C, Smith S, et al. (1994) A controlled study of a stress-reduction, cognitive-behavioral treatment program in fibromyalgia. J Musculoskelet Pain 4: 53-66.

16. Hoffman BM, Papas RK, Chatkoff DK, Kerns RD (2007) Meta-analysis of psychological interventions for chronic low back pain. Health Psychol 26: 1-9.

17. Holroyd KA, Nash JM, Pingel JD, Cordingley GE, Jerome A (1991) A comparison of pharmacological (amitriptyline $\mathrm{HCL}$ ) and nonpharmacological (cognitive-behavioral) therapies for chronic tension headaches. J Consult Clin Psychol 59: 387-393.

18. Keefe FJ, Caldwell DS, Williams DA, Gil KM, Mitchell D, et al. (1990) Pain coping skills training in the management of osteoarthritic knee pain: $A$ comparative study. Behav Ther 21: 49-62.

19. Lamb S, Hansen Z, Lall R, Castelnuovo E, Withers E, et al. (2010) Group cognitive behavioral treatment for low-back pain in primary care: A randomized controlled trial and cost-effectiveness analysis. Lancet 375: 916-923.

20. Morley S, Eccleston C, Williams A (1999) Systematic review and meta-analysis of randomized controlled trials of cognitive behaviour therapy and behaviour therapy for chronic pain in adults, excluding headache. Pain 80: 1-13.

21. Turner JA, Clancy S, McQuade KJ, Cardenas DD (1990) Effectiveness of behavioral therapy for chronic low back pain: a component analysis. J Consult Clin Psychol 58: 573-579.

22. Cosio D (2015) Practice-based evidence for outpatient, acceptance \& commitment versus cognitive-behavioral group therapies for veterans with chronic, non-cancer pain. J Contextual Behav Sci.

23. Duigan N, Burke A (2010) Group based treatment for chronic pain: Is ACT effective and how does it compare to CBT? Poster session presented at Association for Contextual Behavioral Science, $4^{\text {th }}$ Australian and New Zealand Conference of Acceptance and Commitment Therapy, Adelaide, Australia.

24. Vowles K, Wetherell J, Sorrell J (2008) Targeting acceptance, mindfulness, and values-based action in chronic pain: Findings of two preliminary trials of an outpatient group-based intervention. Cogn Behav Pract 16: 49-58.

25. Wetherell JL, Afari N, Rutledge T, Sorrell JT, Stoddard JA, et al. (2011) A randomized, controlled trial of acceptance and commitment therapy and cognitive-behavioral therapy for chronic pain. Pain 152: 2098-2107.

26. Davidson MA, Tripp DA, Fabrigar LR, Davidson PR (2008) Chronic pain assessment: a seven-factor model. Pain Res Manag 13: 299-308.

27. Kazdin AE (2003) Research design in clinical psychology (4 ${ }^{\text {th }}$ edn.) Boston, MA: Allyn and Bacon.

28. Beck AT, Epstein N, Brown G, Steer RA (1988) An inventory for measuring clinical anxiety: psychometric properties. J Consult Clin Psychol 56: 893-897.

29. Fishman SM, Ballantyne JC, Rathmell JP (2009) Bonica's management of pain ( $4^{\text {th }}$ edn.) Baltimore.

30. Winterowd C, Beck AT, Gruener D (2003) Cognitive therapy with chronic pain patients. New York: Springer Publishing Company.

31. Gibbons CJ, DeRubeis RJ (2008) Anxiety symptom focus in sessions of cognitive therapy for depression. Behav Ther 39: 117-125.

32. Barlow DH, Hayes SC, Nelson RO (1984) The scientist practitioner: Research and accountability in clinical and educational settings. New York: Pergamon Press.

33. Gironda RJ, Clark ME, Massengale JP, Walker RL (2006) Pain among veterans of Operations Enduring Freedom and Iraqi Freedom. Pain Med 7: 339-343.

34. Kerns RD, Otis J, Rosenberg R, Reid MC (2003) Veterans' reports of pain and associations with ratings of health, health-risk behaviors, affective distress, and use of the healthcare system. J Rehabil Res Dev 40: 371-379.

35. Drake D, Beckworth W, Brown R, McNeary L, Cifu D, et al. (2006) A profile of patients in a VA pain clinic. Fed Pract 23: 15-22.

36. Dunphy RC, Bridgewater L, Price DD, Robinson ME, Zeilman CJ 3rd, et al (2003) Visceral and cutaneous hypersensitivity in Persian Gulf war veterans with chronic gastrointestinal symptoms. Pain 102: 79-85.

37. Engel CC, Jaffer A, Adkins J, Riddle JR, Gibson R (2004) Can we prevent a second 'Gulf War syndrome'? Population-based healthcare for chronic idiopathic pain and fatigue after war. Adv Psychosom Med 25: 102-122.

38. Ford JD, Campbell KA, Storzbach D, Binder LM, Anger WK, et al. (2001) Posttraumatic stress symptomatology is associated with unexplained illness attributed to Persian Gulf War military service. Psychosom Med 63: 842-849.

39. Kerns RD, Philip EJ, Lee AW, Rosenberger PH (2011) Implementation of the veterans health administration national pain management strategy. Trans Behav Med 1: 635-643.

40. Cosio D, Lin EH (2013) Effects of a pain education program for veterans with chronic, noncancer pain: a pilot study. J Pain Palliat Care Pharmacother 27 340-349.

41. Carnes D, Homer KE, Miles CL, Pincus T, Underwood M, et al. (2012) Effective delivery styles and content for self-management interventions for chronic musculoskeletal pain: a systematic literature review. Clin J Pain 28: 344-354.

42. Lappalainen R, Lehtonen T, Skarp E, Taubert E, Ojanen M, et al. (2007) The impact of CBT and ACT models using psychology trainee therapists: a preliminary controlled effectiveness trial. Behav Modif 31: 488-511.

43. Otis $\mathrm{J}$ (2007) Managing chronic pain: A cognitive-behavioral therapy approach therapist guide (treatments that work). New York: Oxford University Press.

44. Dahl J, Wilson K, Luciano C, Hayes S (2005) Acceptance and commitment therapy for chronic pain. Reno, NV: Context Press.

45. Vowles K, Sorrell J (2007) Life with chronic pain: An acceptance-based approach. Therapist guide and patient workbook. University of Virginia, and San Mateo Medical Center and VA Palo Alto Health Care System, Center for Health Care Evaluation.

46. Dahl J, Hayes SC, Lundgren T (2006) Living beyond your pain: Using acceptance \& commitment therapy to ease chronic pain. Oakland, CA: New Harbinger Publications, Inc.

47. http://painconsortium.nih.gov/pain_scales/NumericRating Scale.pdf

48. Derogatis LR (2000) BSI-18 administration, scoring and procedures manual. Minneapolis, MN: National Computer Systems.

49. Osman A, Kopper BA, Barrios FX, Osman JR, Wade T (1997) The Beck Anxiety Inventory: reexamination of factor structure and psychometric properties. J Clin Psychol 53: 7-14.

50. Piotrowski C (1999) The status of the Beck Anxiety Inventory in contemporary research. Psychol Rep 85: 261-262.

51. Blankers M, Koeter MW, Schippers GM (2010) Missing data approaches in eHealth research: simulation study and a tutorial for nonmathematically inclined researchers. J Med Internet Res 12: e54.

52. Streiner D, Geddes J (2001) Intention to treat analysis in clinical trials when there are missing data. Evid Based Ment Health 4: 70-71.

53. http://biostat.mc.vanderbilt.edu/wiki/Main/PowerSampleSize.

54. Borden JW, Peterson DR, Jackson EA (1991) The Beck Anxiety Inventory in nonclinical samples: Initial psychometric properties. J Psychopathol Behav Assess 13: 345-356

55. Tabachnick BG, Fidell LS (2001) Using multivariate statistics (4 ${ }^{\text {th }}$ edn.) Boston, MA: Allyn and Bacon.

56. Kaiser HF (1970) A second generation little jiffy. Psychometrika 35: 401-415

57. Kaiser HF (1974) An index of factorial simplicity. Psychometrika 39: 31-36

58. Bartlett MS (1954) A note on multiplying factors for various chi-squared approximations. J R Stat Soc 16: 296-298.

59. O'Connor BP (2000) SPSS and SAS programs for determining the number of components using parallel analysis and velicer's MAP test. Behav Res Methods Instrum Comput 32: 396-402.

60. Howell DC (2002) Statistical methods for psychology (5 $5^{\text {th }}$ edn.) Belmont, CA Duxbury Press.

61. Burns JW, Nielson WR, Jensen MP, Heapy A, Czlapinski R, et al. (2015) Specific and general therapeutic mechanisms in cognitive behavioral treatment of chronic pain. J Consult Clin Psychol 83: 1-11.

62. Forman EM, Herbert JD, Moitra E, Yeomans PD, Geller PA (2007) A randomized controlled effectiveness trial of acceptance and commitment therapy and cognitive therapy for anxiety and depression. Behav Modif 31: 772-799. 
Citation: Cosio D, Swaroop S (2016) The Use of Mind-body Medicine in Chronic Pain Management: Differential Trends and Session-by-Session Changes in Anxiety. J Pain Manage Med 2: 114.

63. Hall PA, Fong GT (2010) Temporal self-regulation theory: Looking forward. Health Psychol Rev 4: 83-92.

64. Gross JJ, John OP (2003) Individual differences in two emotion regulation processes: implications for affect, relationships, and well-being. J Pers Soc Psychol 85: 348-362.

65. McCracken LM, Gutiérrez-Martínez O (2011) Processes of change in psychological flexibility in an interdisciplinary group-based treatment for chronic pain based on Acceptance and Commitment Therapy. Behav Res Ther 49: 267-274.

66. Hayes SA, Miller NA, Hope DA, Heimberg RG, Juster HR (2008) Assessing Client Progress Session by Session in the Treatment of Social Anxiety Disorder The Social Anxiety Session Change Index. Cogn Behav Pract 15: 203-2011.

67. http://www.va.gov/vetdata/docs/SpecialReports/Profile_of_Veterans_2009_ FINAL.pdf. 